Earth science

\section{Just add more water}

Rob Evans

Data from a different technique for probing events within the Earth, interpreted in terms of a new hypothesis about the effects of water at depth, raise tantalizing questions about recycling of a tectonic plate.

n places such as the Pacific Rim, the sea floor collides with and is pushed below the land masses that border the ocean. This process of subduction, and its consequences in the form of earthquakes and volcanism, are the result of plate tectonics, in which the formation of sea floor at mid-ocean ridges is accommodated by the recycling of tectonic plates in the Earth's interior. But the recycling is a complex process: the interaction of the subducting plate, or slab, with the surrounding material itself produces volcanism and, in some settings, new sea floor. On page 399 of this issue, Booker et al. ${ }^{1}$ present dramatic models of the subduction system beneath the Andes that suggest that the recycling processes run deeper than previously thought. Their interpretation depends on new data, which in themselves are striking but which also allow the authors to make some inferences about the influence of water at depth.

Water plays an important role in subduction recycling: its release from the oceanic plate can affect the rheology of the rock as it moves into the mantle, below the Earth's crust, and also increases rock melting ${ }^{2}$. Yet the amount of water released, the depth of release and the pathways it takes are poorly defined, as are the depths of melting and the route that the melt takes as it moves from close to the subducting plate to its eruption in the overlying volcanic arc.
Seismic techniques for looking into the Earth will be familiar to most people. But there are other approaches, one being the magnetotelluric (MT) method, which offers promise for locating melt and identifying the distribution of water in the mantle. This technique uses naturally occurring electric currents in the ionosphere, created by the capture of charged particles by the planet's magnetic field, to measure Earth's electrical conductivity. That conductivity depends partly on composition and temperature. But it can be dramatically increased by small amounts of partial melt, provided that the melt forms an interconnected network ${ }^{3}$. And it can also be affected by water in the mantle, in the form of dissolved hydrogen, both above and below the transition that occurs globally at around $410 \mathrm{~km}$, where the mantle composition undergoes a change from one mineral phase (olivine $e^{4}$ ) to another (wadsleyite ${ }^{5}$ ). MT has been used only infrequently to address subduction-zone processes, mostly because these systems are close to the ocean and require complex onshore-offshore investigations ${ }^{6,7}$. Not only are the results of Booker et al. ${ }^{1}$ dramatic in their own right, they also represent a significant advance in MT imaging of subduction systems.

Where Booker et al. have made their measurements, the subducting slab flattens beneath South America before plunging down into the mantle. This makes the area somewhat anomalous - an inactive volcanic arc sits about $200 \mathrm{~km}$ above the slab, instead of the more normal $100 \mathrm{~km}$. But it does mean that the key subduction processes occur farther inland beneath the continent, and can be imaged using terrestrial MT (see Fig. 1 of the paper on page 400).

The most significant part of Booker and colleagues' model is the conductive anomaly that appears to coincide with the top of the subducting slab. The introduction of fluids from the slab, resulting in melting, seems one obvious explanation for the anomaly. Yet what is surprising is the great depth - at least $250 \mathrm{~km}$ but perhaps as much as $400 \mathrm{~km}$ — to which this melting seems to be occurring. Earthquake activity from the adjacent slab (there is very little activity in the region sampled by Booker et al.) shows intermediate-depth earthquakes ending around 200 $\mathrm{km}$, and it has been suggested that this marks the depth limit of water-bearing mineral phases $^{8,9}$. In fact, the MT data indicate that there is electrical conductivity along or just above the flat part of the slab (above $200 \mathrm{~km}$ ), suggesting some water loss at these depths.

There are few other clues that can help to account for this apparent deeper melting. Analyses of samples from an overlying inactive volcano, Pocho, are equivocal, because much of the chemistry of the samples reflects processes occurring in the crust ${ }^{10}$; in any event, their age means that they might easily reflect a different melting geometry. The prolonged passage of an old slab under the continent at relatively shallow depths is unusual, and might provide an explanation for why the slab has been able to hold on to its water to greater depths than usual. Perhaps it results in the breakdown of mineral phases that do not normally contribute to the water budget.

Organic chemistry

\title{
000 !
}

In an organic molecule, how long can a chain of oxygen atoms be? No chain-like polyoxides are known that have more than three oxygen atoms in a row; only a few trioxides are known. But to this collection, Holger Pernice et al. now add bis(fluoroformyl)trioxide, or FC(0)000C(0)F (Angew. Chem. Int. Edn 43, 2843-2846; 2004). Bis(fluoroformyl)trioxide is an acyl compound, characterized by a $\mathrm{C}=0$ acid-group derivative. It is the second known example of a chainlike acyl trioxide (after the more complex $\left.\mathrm{CF}_{3} \mathrm{OC}(0) 000 \mathrm{C}(0) 0 \mathrm{CF}_{3}\right)$ but the first to have its twisting, chiral structure determined.
Starting from a potentially explosive mixture of carbon monoxide, fluorine and oxygen, the reaction proceeds to produce the acyl trioxide and the peroxide $\mathrm{FC}(0) 00 \mathrm{C}(0) \mathrm{F}$. The spectrum of infrared radiation absorbed by the sample has bands at around 800 and $900 \mathrm{~cm}^{-1}$ that are typical of 0-0-0 stretching vibrations. Similarly, data from ultraviolet, nuclear magnetic resonance and $X$-ray diffraction studies confirm the formation of the trioxide.

For the determination of its structure, Pernice et al. allowed a trioxide sample to crystallize at a temperature of $-78^{\circ} \mathrm{C}$ in a stream of nitrogen gas. Diffraction data show that the molecule has a trans-syn-syn structure - that is, the $\mathrm{C}-\mathrm{O}$ bonds are in the trans (opposite) conformation with respect to the plane of the three bonded oxygen atoms, and the two $\mathrm{C}=0$ bonds are in the syn (same-side) conformation. The left-handed version of this structure is shown here (oxygen in red, carbon in blue, fluorine in green).

Theory predicts that another structure, or 'rotamer', should exist: trans-syn-anti, in which, instead of curling round, the molecule

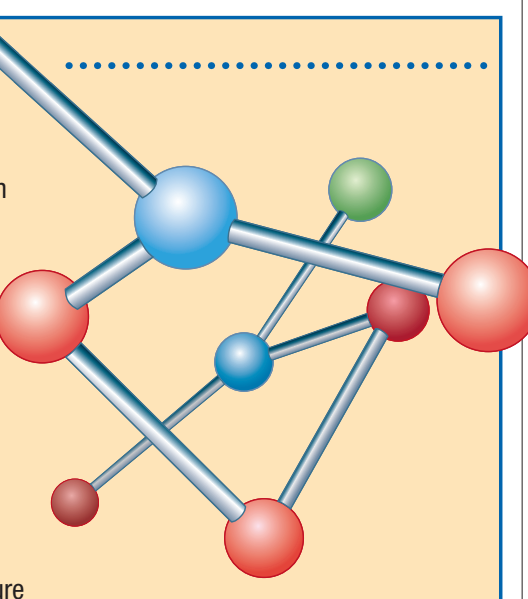

twists back on itself about the central oxygen atom. Pernice et al. say that their infrared data indeed suggest the presence of a second rotamer.

Alison Wright 
Booker et al. ${ }^{1}$ explain the deep melting by invoking the ideas of Bercovici and Karato ${ }^{11}$. This new hypothesis suggests that a layer of melt exists just above the $410-\mathrm{km}$ boundary as slowly upwelling mantle undergoes a change from water-rich wadsleyite to olivine, liberating water and inducing melting. In Booker and colleagues' models, the melt column seems to originate as much from the $410-\mathrm{km}$ boundary as it does from the subducting slab, and so the suggestion of a link between the two is not surprising. The authors propose that the liberation of additional water from the slab induces further production of buoyant melt, a necessary condition if the melt is to rise as is seen.

This link to Bercovici and Karato's model is intriguing, but I'm not completely sold on it. Undoubtedly, the argument would be strengthened by better data on the structure across the 410-km boundary which, as the authors point out, is not the best-resolved feature of the model. Booker et al. suggest that the mantle to the west of (or beneath) the slab is dehydrated as a result of melt extraction at the East Pacific Rise, part of the mid-ocean ridge system in the Pacific Ocean. Although this might be true for the upper $60-80 \mathrm{~km}$ or so of oceanic plate from which melt and water have been extracted, other data suggest that there is plenty of remaining water that can increase electrical conductivity in the mantle below about $80 \mathrm{~km}$ depth ${ }^{12}$. This means that only the region adjacent to the subducting slab would be expected to be dry. If there is water around, we would expect a more or less uniform increase in conductivity at $410 \mathrm{~km}$ across the region. Although their models show a stepwise increase across the $410-\mathrm{km}$ boundary, Booker et al. point out that the data are consistent with a flat $410-\mathrm{km}$ transition throughout the region. Why is all this important? Well, it speaks to outstanding issues in terms of resolution of this critical part of the system.

Regardless of the details of the 410-km boundary, the authors' primary observation - an electrical conductor that must surely represent a subduction-related melt column rising from depth - is striking. And, as they point out, issues pertaining to the $410-\mathrm{km}$ boundary and the link to the BercoviciKarato hypothesis can be addressed with measurements made with a longer chain of MT stations. If the interaction with a melt layer at $410 \mathrm{~km}$ is the explanation, it should be seen in other subduction systems. It hasn't been seen elsewhere yet, but maybe we just need to look more carefully.

Rob Evans is at Woods Hole Oceanographic

Institution, Woods Hole, Massachusetts 02543, USA. e-mail:revans@whoi.edu

1. Booker, J. R., Favetto, A. \& Pomposiello, M. C. Nature 429, 399-403 (2004).

2. Hirth, G. \& Kohlstedt, D. L. Earth Planet. Sci. Lett. 144, 93-108 (1996).

3. Roberts, J. J. \& Tyburczy, J. A. J. Geophys. Res. 104, 7055-7066 (1999).

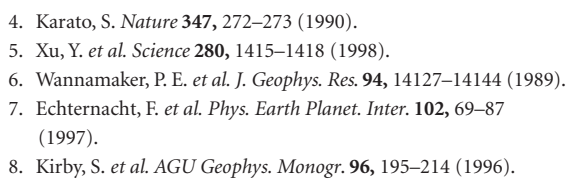

9. Hacker, B. R. et al. J. Geophys. Res. 108, doi:10.1029/2001JB001129 (2003).

10. Kay, S. M. \& Gordillo, C. E. Contrib. Mineral. Petrol. 117, 25-44 (1994).

11. Bercovici, D. \& Karato, S. Nature 425, 39-44 (2003).

12. Lizarralde, D. et al. J. Geophys. Res. 100, 17837-17854 (1995).
M into usable energy forms. To meet demands, every cell contains thousands of them. Unlike most other cellular compartments, mitochondria have their own genomes, which encode a few mitochondrial proteins (most others being encoded by genes in the nucleus). In numerous non-reproductive tissues of many species, mitochondrial genes (like nuclear genes) accumulate mutations as the animals age ${ }^{1}$, and it has been speculated that these mutations might in fact cause ageing, by leading to energy-generation defects - increased numbers of harmful reactive oxygen species, cellular damage and so on. On the other hand, the association between mitochondrial mutations and ageing could merely be correlative - these mutations might simply be one of the manifestations of growing old. That possibility becomes less likely, however, with the publication of the paper on page 417 of this issue by Trifunovic and colleagues ${ }^{2}$.

Point mutations (single base-pair changes $)^{3}$, deletions and rearrangements ${ }^{4}$ in mitochondrial DNA accumulate in several non-reproductive (somatic) tissues during ageing. To find out whether such alterations are a cause or a correlate of growing old, Trifunovic et al. ${ }^{2}$ genetically engineered mice to carry mutations in an enzyme called DNA polymerase- $\gamma$. Encoded by nuclear genes and transported to mitochondria, this protein is believed to be responsible for all aspects of mitochondrial DNA metabolism: it both copies and proofreads the DNA, eliminating errors it makes during replication, and it is also believed to participate in the resynthesis of DNA during DNA-repair processes. New mitochondria replace old mitochondria in all cell types throughout life, and new mitochondria must also be made when cells divide. These events require the replication of mitochondrial DNA.

Trifunovic and colleagues wanted to render the mitochondrial DNA polymerase error-prone by eliminating its proofreading activity while maintaining its catalytic potency - the rationale being that any errors in mitochondrial DNA replication would go unnoticed by the cell, and so mutations would accumulate. As the mice would have this error-prone polymerase from youth, it would be possible to see whether or not the mutations it produces accelerate ageing. To eliminate the proofreading activity, the authors substituted an alanine amino acid for a crucial aspartate in the relevant region of the enzyme molecule.

They found that the somatic tissues of mice bearing two mutant copies of the DNA polymerase- $\gamma$ gene indeed showed extensive mitochondrial DNA mutations, largely comprising deletions and point mutations. The percentage of mitochondria bearing deletions was similar in different tissues, and did not vary with age, suggesting that the deletions had occurred early in development.

\begin{tabular}{|ll|}
\hline Mutated protein & Effects \\
\hline Lamin A/C (ref. 11) & Defective inner nuclear membrane \\
\hline Ku86, XPD (refs 12, 13) & Defective metabolism of nuclear DNA \\
\hline DNA polymerase- $\gamma$ (ref. 2) & Defective metabolism of mitochondrial DNA \\
\hline Telomerase (ref.14) & Defective regulation of chromosome caps \\
\hline p53 (ref. 15) & Altered regulation of cell-division cycle and cell death \\
\hline Klotho (ref. 16) & Impaired calcium and vitamin D metabolism? \\
\hline
\end{tabular}

Figure 1 Many mutational pathways can accelerate 'segmental ageing' (or, to use more conservative nomenclature, segmental ageing-like syndromes ${ }^{10}$ ) in mice. Published literature ${ }^{2,11-16}$ suggests that there may be at least six pathways that generate partially overlapping subsets of features consistent with accelerated ageing. Several of these pathways are likely to interact ${ }^{17}$, making such classifications problematic. Trifunovic and colleagues' findings ${ }^{2}$, however, provide strong direct support for the mitochondrial pathway. 